\title{
Nanoparticle-Mediated Drug Delivery
}

National Cancer Institute

\section{Source}

National Cancer Institute. Nanoparticle-Mediated Drug Delivery. NCI Thesaurus. Code C62311.

This method entails the attachment of a drug to the surface of nanoparticles. The role of the nanoparticle is to facilitate delivery of the drug to a cellular target that it would not reach in its free form. 